\title{
Comprehensive analysis of differentially expressed IncRNAs as diagnostic and prognostic markers for colorectal cancer
}

\author{
XUNLEI ZHANG ${ }^{1 *}$, XINGSONG ZHANG ${ }^{2 *}$, LILI SHEN $^{3 *}$, LI SONG $^{1}$, JINDONG WU ${ }^{4}$, \\ GUANGXIN CAO ${ }^{4}, \mathrm{XIN} \mathrm{CHEN}^{4}$ and $\mathrm{BIN} \mathrm{ZHU}^{5}$
}

\author{
Departments of ${ }^{1}$ Oncology and ${ }^{2}$ Pathology, Nantong Tumor Hospital, Nantong, Jiangsu 226300; ${ }^{3}$ Department of Oncology, \\ Haimen People's Hospital, Nantong, Jiangsu 226100; ${ }^{4}$ Department of General Surgery, Nantong \\ Tumor Hospital, Nantong, Jiangsu 226300; ${ }^{5}$ Department of General Surgery, Nanjing First \\ Hospital, Nanjing Medical University, Nanjing, Jiangsu 210006, P.R. China
}

Received October 22, 2018; Accepted August 2, 2019

DOI: $10.3892 /$ etm.2019.8067

\begin{abstract}
Colorectal cancer (CRC) is the third most common type of cancer worldwide. Recent studies had revealed the important roles of long non-coding RNAs (lncRNAs) in a variety of human cancers, including CRC. However, the molecular mechanisms associated with CRC remain largely undetermined. In the current study, the GSE21510 dataset was analyzed to identify differentially expressed mRNAs and lncRNAs in CRC samples. The Database for Annotation, Visualization and Integrated Discovery was used to perform Gene Ontology and Kyoto Encyclopedia of Genes and Genomes pathway bioinformatics analysis. Furthermore, protein-protein interaction networks were constructed to reveal interactions among differentially expressed proteins. Kaplan-Meier analysis was subsequently performed to determine the association between key lncRNA expression and the overall survival of patients with CRC. A total of 107 upregulated lncRNAs and 43 downregulated lncRNAs were identified in CRC. A IncRNA mediated co-expression network was also constructed in CRC. Bioinformatics analysis indicated that lncRNAs were associated with a series of biological processes, including 'xenobiotic glucuronidation', 'rRNA processing', 'sister chromatid cohesion', 'cell proliferation', 'mitotic nuclear division' and 'cell
\end{abstract}

Correspondence to: $\mathrm{Dr}$ Xin Chen, Department of General Surgery, Nantong Tumor Hospital, 30 Tongyang Road, Nantong, Jiangsu 226300, P.R. China

E-mail: dcchenxin@yeah.net

Dr Bin Zhu, Department of General Surgery, Nanjing First Hospital, Nanjing Medical University, 68 Changle Road, Nanjing, Jiangsu 210006, P.R. China

E-mail: 477750911@qq.com

*Contributed equally

Key words: long non-coding RNA, prognostic markers, expression profiling, protein-protein interaction analysis, colorectal cancer cycle regulation'. Furthermore, a higher expression of small nucleolar RNA host gene 17, tetratricopeptide repeat domain 2B-antisense RNA (AS) 1, erythrocyte membrane protein band 4.1 like 4A-AS2, deleted in lymphocytic leukemia 2, and a lower expression of muscle blind like splicing regulator 1-AS1 and LOC389332 were associated with shorter overall survival time in CRC samples. The present study provides useful information that can be used in the identification of novel biomarkers for CRC.

\section{Introduction}

Long non-coding RNAs (lncRNAs) are a major class of non-coding RNAs with $>200$ base pairs $(1,2)$. Recent studies have revealed the important roles of IncRNAs in cancer progression (3-6). A variety of IncRNAs have been observed to be dysregulated in a number of human cancers, serve as oncogenes or serve a tumor suppressor role $(7,8)$. LncRNAs are involved in the regulation of a variety of biological processes, including cell growth, apoptosis and differentiation via protein interaction, sponging microRNAs (miRNAs/miRs) and binding to DNA (9-11). For example, lncRNA GATA binding protein 6 -antisense RNA (AS) has been indicated to interact with lysyl oxidase like 2 to transcriptionally regulate gene expression (12). LncRNA associated with BCBM has been revealed to regulate interleukin (IL)-6-triggered signal transducer and activator of transcription 3 phosphorylation by binding to janus kinase 2 (13). Furthermore, lncRNAs have been demonstrated to have a prognostic value of in human cancers. The upregulation of serine peptidase inhibitor kunitz type 1-antisense RNA 1 predicted a poor prognosis in colorectal cancer (14), and the overexpression of H19 imprinted maternally expressed transcript predicted a poor prognosis in melanoma (15). These results demonstrated that lncRNAs serves a crucial role in regulating human cancer progression.

Colorectal cancer (CRC) is the third most common type of cancer worldwide (16). Worldwide, the morbidity and mortality of CRC has increased rapidly in recent years, with 134,490 new cases and 49,190 cases of CRC-associated mortality in 2016 (17). Previous studies have indicated that lncRNAs are 
associated with CRC progression and prognosis. IncRNA BRAF non-coding RNA was demonstrated to promote $\mathrm{CRC}$ tumorigenesis and enhance adriamycin resistance, and LINC00312 was indicated to repress CRC cell proliferation and metastasis via miR-21 $(18,19)$. However, the expression pattern and functional roles of a large number of lncRNAs remain undetermined. Therefore, there is an urgent requirement for the identification of CRC-associated lncRNAs and for the generation of useful information that can be used to understand their potential roles in CRC.

With the development of microarray profiles and RNA sequencing, a series of public gene expression analyses were published, including The Cancer Genome Atlas (TCGA; https://portal.gdc.cancer.gov/) and Gene Expression Omnibus (GEO; https://www.ncbi.nlm.nih.gov/gds) datasets. These databases provide powerful tools that can be used to identify cancer-associated mRNAs, miRNAs and lncRNAs. For example, Zhang et al (20) identified 218 differently expressed genes in in papillary thyroid cancer using a TCGA dataset. Yuan et al (21) identified 1,176 lncRNAs and 245 miRNAs in colorectal cancer using the TCGA dataset. A number of lncRNAs, which were predicted using bioinformatics, were identified to be potential biomarkers in a variety of human cancer types. A total of four lncRNAs (LINC01018, LOC553137, miR4435-2HG and TTTY14) were revealed to be correlated with overall survival in patients with gastric cancer (22). An increased expression of miR100HG has also been associated with poor prognosis in patients with breast cancer (23). Emerging evidence has indicated bioinformatics analyses to be a powerful method that can be used to understand the potential roles of novel lncRNAs in a variety of human diseases, including cancer. Wang et al (24) demonstrated that dysregulated lncRNAs in esophageal squamous cell carcinoma were associated with the regulation of cancer metastasis, using co-expression analysis (24). Cao et al (25) also demonstrated that dysregulated lncRNAs in bladder cancer were associated with the regulation of cell migration and the Ras signaling pathway.

In the present study, a GEO dataset GSE21510 was re-annotated to identify CRC associated mRNAs and lncRNAs. Bioinformatics analyses were also performed to understand the potential roles of these lncRNAs in CRC. The current study aimed to provide a novel insight into CRC-associated lncRNAs that could serve as biomarkers in CRC.

\section{Materials and methods}

LncRNA classification pipeline. A pipeline, which was previously described by Zhang et al (26), was used to re-annotate microarray data.

Microarray data and data preprocessing. In the present study, GSE21510 dataset (27) was downloaded from the GEO database to identify differently expressed mRNAs and lncRNAs. GSE21510 included 25 normal and 123 CRC samples. A total of 104 patients were assigned to the microarray study, including 13 patients with stage I, 37 patients with stage II, 34 patients with stage III and 20 patients with stage IV CRC. Metastatic recurrence after surgery occurred in 18 patients with stage I-III disease, and the median follow-up time was
22 months for these patients. The raw data were normalized using robust multi-array average (RMA) method (28) using R 2.6.2 statistical software (29) with the affy package (30) from BioConductor V3.9 (http://www.bioconductor.org/). Normalization was separately performed for LCM dataset and homogenized tissue dataset (31). The normalized gene expression levels were presented as log2-transformed values using the RMA method. LncRNAs or mRNAs with fold changes (FC) $\geq 2$ and $\mathrm{P}<0.05$ were considered as differentially expressed lncRNAs or mRNAs. Furthermore, gene expression profile data in CRC (including 275 colon adenocarcinoma samples and 92 rectum adenocarcinoma samples) and normal tissues were downloaded from TCGA (https://portal.gdc.cancer.gov/) and used to examine the expression pattern of key lncRNA in $\mathrm{CRC}$ and its association with the patient clinicopathological features.

Functional group analysis. The Database for Annotation, Visualization and Integrated Discovery v6.8 (http://david. ncifcrf.gov/) was used to perform Gene Ontology (GO) and Kyoto Encyclopedia of Genes and Genomes (KEGG) pathway enrichment analyses. GO analyses included biological process, cellular component and molecular function. GO terms and KEGG pathways with a value of $\mathrm{P}<0.05$ were considered to be significantly enriched functional annotations.

lncRNA-mRNA correlation network. The lncRNA-mRNA correlation network was established according to IncRNA target predictions along with differentially expressed lncRNA and mRNA profiles in GSE21510. A total of 3,647 lncRNA-mRNA pairs with an absolute Pearson value $>0.85$ were selected to construct the co-expression network in CRC.

Statistical analysis. Statistical comparisons between groups were performed using a t-test or Mann-Whitney U-test, as appropriate. Kaplan-Meier survival curves were constructed with the log-rank test and P-values were calculated. The cut-off threshold was set to $\mathrm{P}<0.05$. A log-rank test was conducted to examine the significance of the difference between the curves. $\mathrm{P}<0.05$ was considered to indicate a statistically significant result, with a $95 \%$ confidence level.

\section{Results}

Identification of the differentially expressed mRNAs and lncRNAs in CRC. A public GEO dataset, GSE21510, which included 25 normal and 123 CRC samples was analyzed to identify CRC-associated mRNAs and lncRNAs. A total of 2,387 mRNAs were revealed to be differentially expressed in CRC with a fold change of $>1.5$ and $P<0.01$. Among these mRNAs, 1,874 were upregulated and 1,385 were downregulated in CRC compared with normal samples. Hierarchical clustering was used to identify differentially expressed mRNAs in CRC (Fig. 1A).

After re-annotating GSE21510 according to Zhang et al (26) report, a total of 107 upregulated lncRNAs and 43 downregulated lncRNAs were identified in CRC compared with normal samples with a fold change of $>1.5$ and $\mathrm{P}<0.01$. Among these genes, a number of lncRNAs, including colorectal neoplasia differentially expressed (CRNDE) (32), 

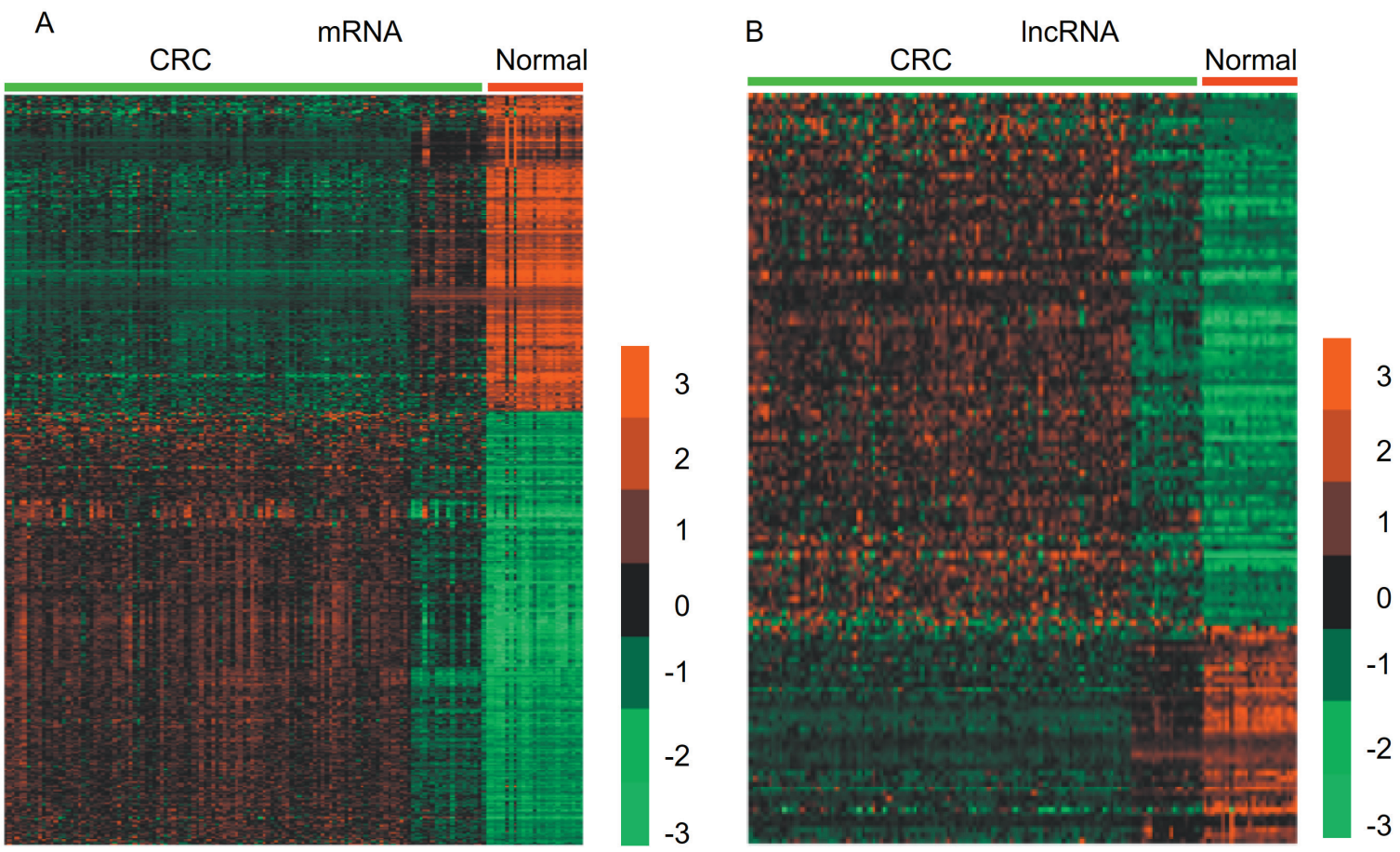

Figure 1. Identification of the differentially expressed mRNAs and lncRNAs in CRC. Hierarchical clustering analysis indicating the differential (A) mRNA expression and (B) lncRNA expression in CRC, using GSE21510. Red indicates high expression and green indicates low expression. 3,2, 1, 0, -1, -2, and -3 are fold changes compared with normal samples. lncRNA, long non-coding RNA; CRC, colorectal cancer; normal, normal tissue samples.

growth arrest specific 5 (GAS5) (33), small nucleolar RNA host gene 1 (SNHG1) (34) and, ZNFX1 antisense RNA 1 (ZFAS1) (35) have been identified to be associated with CRC progression in previous studies (36-38). However, most lncRNAs, including LINC00525, LINC00869 and LINC01279, have not been previously identified in human cancers and still need to be investigated further. The present study identified that CRNDE, deleted in lymphocytic leukemia 2 (DLEU2) and LOC101060264 were revealed to be upregulated most, and LOC101929340, CDKN2B-AS1 and LOC101928405 lncRNAs were revealed to be downregulated most in CRC. Hierarchical clustering was used to present differentially expressed lncRNAs in CRC (Fig. 1B).

\section{Construction of lncRNAs}

co-expression network in CRC. The differentially expressed IncRNA co-expression network in CRC was subsequently constructed according to expression values in GSE21510. A total of 3,647 lncRNA-mRNA pairs with an absolute Pearson value $>0.85$ were selected to construct the co-expression network in CRC. This network included 47 lncRNAs and 636 mRNAs, as presented in Fig. 2.

A number of IncRNAs were identified as key regulators in CRC. For example, lncRNA LOC389332 was co-expressed with 328 mRNAs, LOC101928405 with 295 mRNAs, FLJ36848 with 260 mRNAs and EPB41L4A-AS2 with 251 mRNAs. A total of 4 mRNAs were co-expressed with $>20$ lncRNAs in CRC, including yip1 interacting factor homolog $\mathrm{B}$ (YIF1B), zinc finger and BTB domain containing 7B (ZBTB7B), ruvb like AAA ATPase 2 (RUVBL2) and vesicle associated membrane protein 2 (VAMP2) (Fig. 2).
Bioinformatics analysis for differentially expressed lncRNAs in $C R C$. Considering that $\operatorname{lncRNAs}$ serves a regulatory role in a number of human cancers through downstream targets, GO and KEGG analysis were subsequently performed to reveal the potential roles of lncRNAs in CRC using their co-expressing mRNAs. GO analysis indicated that differentially expressed lncRNAs were associated with numerous biological processes, including 'xenobiotic glucuronidation', 'rRNA processing', 'sister chromatid cohesion', 'regulation of Rac protein signal transduction', 'cell proliferation', 'mitotic nuclear division', 'negative regulation of cellular glucuronidation', 'regulation of transcription', 'DNA replication', 'keratan sulfate biosynthetic process', 'negative regulation of fatty acid metabolic process', 'telomere maintenance via recombination', 'oligosaccharide biosynthetic process', 'flavonoid glucuronidation' and 'cell cycle' (all, $\mathrm{P}<0.001$; Fig. 3A).

KEGG pathway analysis revealed that differentially expressed lncRNAs were associated with a series of pathways, including 'pentose and glucuronate interconversions', 'glycosphingolipid biosynthesis', 'ascorbate and aldarate metabolism', 'chemical carcinogenesis', 'retinol metabolism', 'mismatch repair', 'bile secretion', 'cell cycle', 'metabolism of xenobiotics by cytochrome P450', 'drug metabolism-other enzymes', p53 signaling pathway, 'drug metabolism-cytochrome P450' and 'ibosome biogenesis in eukaryotes' (all, $\mathrm{P}<0.001$; Fig. 3B).

TCGA dataset validation of expression of key lncRNAs in CRC. As presented in Fig. 4, a total of 16 key lncRNAs were identified to be dysregulated in CRC when compared with normal samples using the TCGA dataset. Among these lncRNAs, programmed cell death 4 (PDCD4)-AS1 $(\mathrm{P}<0.001)$, heart and neural crest 


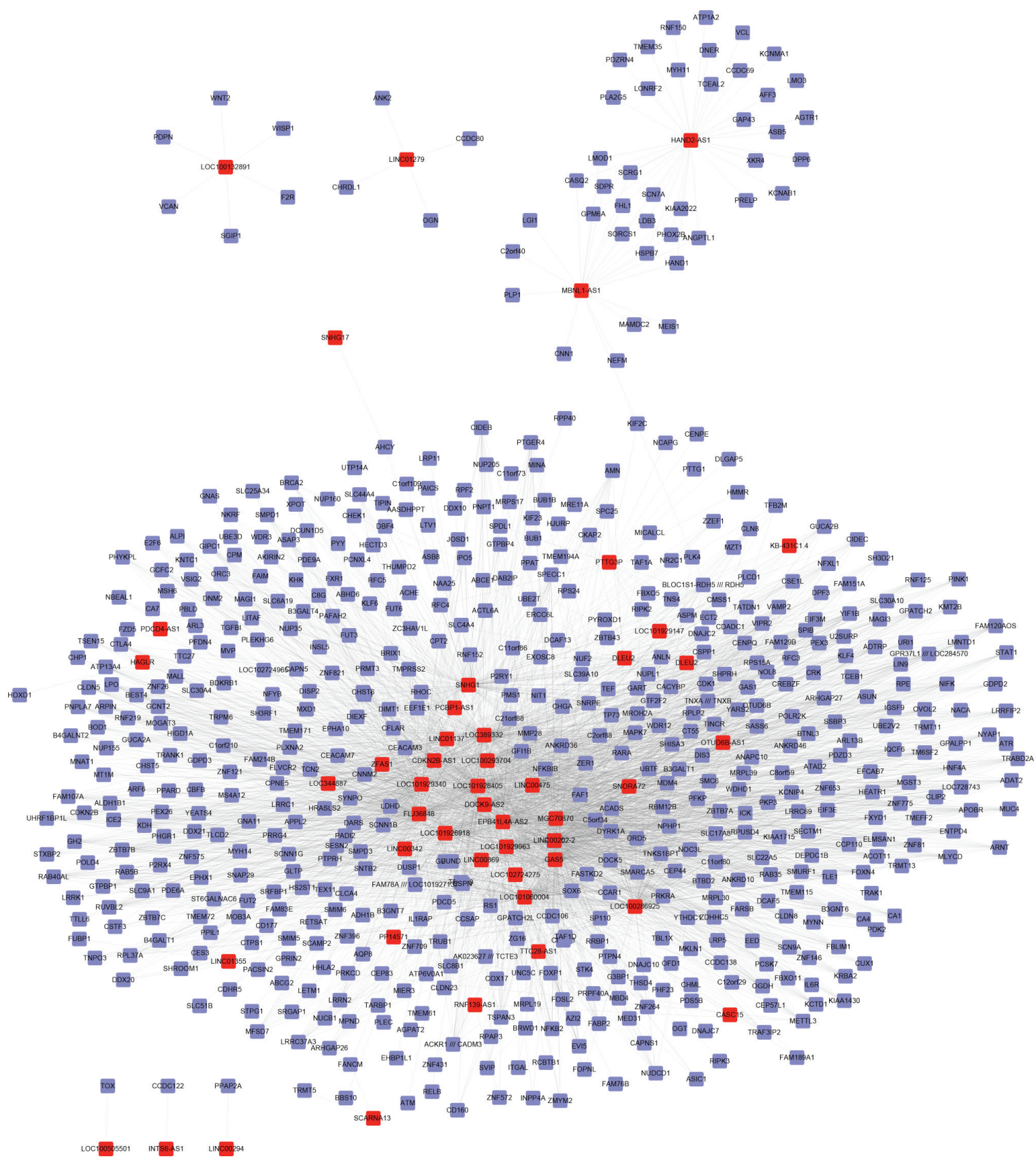

Figure 2. Construction of lncRNAs co-expression network in CRC. The differentially expressed lncRNA co-expression networks in CRC according to their expression levels in GSE21510, indicating a number of lncRNAs as key regulators in CRC. Red node represents lncRNA, and purple node represents mRNA. lncRNA, long non-coding RNA; CRC, colorectal cancer.

derivatives expressed 2 (HAND2)-AS1 ( $\mathrm{P}<0.001)$, erythrocyte membrane protein band 4.1 like 4A(EPB41L4A)-AS2 ( $<<0.001)$, LOC389332 $(\mathrm{P}<0.001)$, long intergenic non-protein coding RNA 294 (LINC00294; $\mathrm{P}<0.001)$ and PP14571 $(\mathrm{P}<0.001)$ were downregulated in CRC in comparison with normal samples. However, LINC00202-2 ( $\mathrm{P}<0.001)$, DLEU2 $(\mathrm{P}<0.001)$, GAS5 $(\mathrm{P}<0.001)$, SNHG1 $(\mathrm{P}<0.001)$, small nucleolar RNA host gene 17 (SNHG17; $\mathrm{P}<0.001$ ), pituitary tumor-transforming 3 pseudogene (PTTG3P; $\mathrm{P}<0.001$ ), muscle blind like splicing regulator 1 (MBNL1)-AS1 $(\mathrm{P}<0.001)$, ZFAS1 $(\mathrm{P}<0.001)$, tetratricopeptide repeat domain 2B (TTC28)-AS1 $(\mathrm{P}<0.001)$ and cancer susceptibility 15 (CASC15; $\mathrm{P}<0.001)$ were upregulated in $\mathrm{CRC}$ when compared with normal tissues.
Dysregulation of key lncRNAs is associated with overall survival time in CRC. Kaplan-Meier analysis was subsequently performed to explore whether key lncRNAs could serve as novel CRC biomarkers. The online Cutoff Finder (39) (http://molpath.charite.de/cutoff/output.jsp) was used to determine the cut-off value to divide all patients with CRC into the lncRNA low and high group. As presented in Fig. 5, higher expression levels of SNHG17 ( $\mathrm{P}=0.0097)$, TTC28-AS1 $(\mathrm{P}=0.0065)$, EPB41L4A-AS2 $(\mathrm{P}=0.0075)$, DLEU2 $(\mathrm{P}=0.007)$ and SNHG1 $(\mathrm{P}=0.0033)$ were associated with shorter overall survival time of patients with CRC compared with the respective low expression groups, whereas the increased expression levels of MBNL1-AS1 $(\mathrm{P}=0.0002)$ and LOC389332 
A

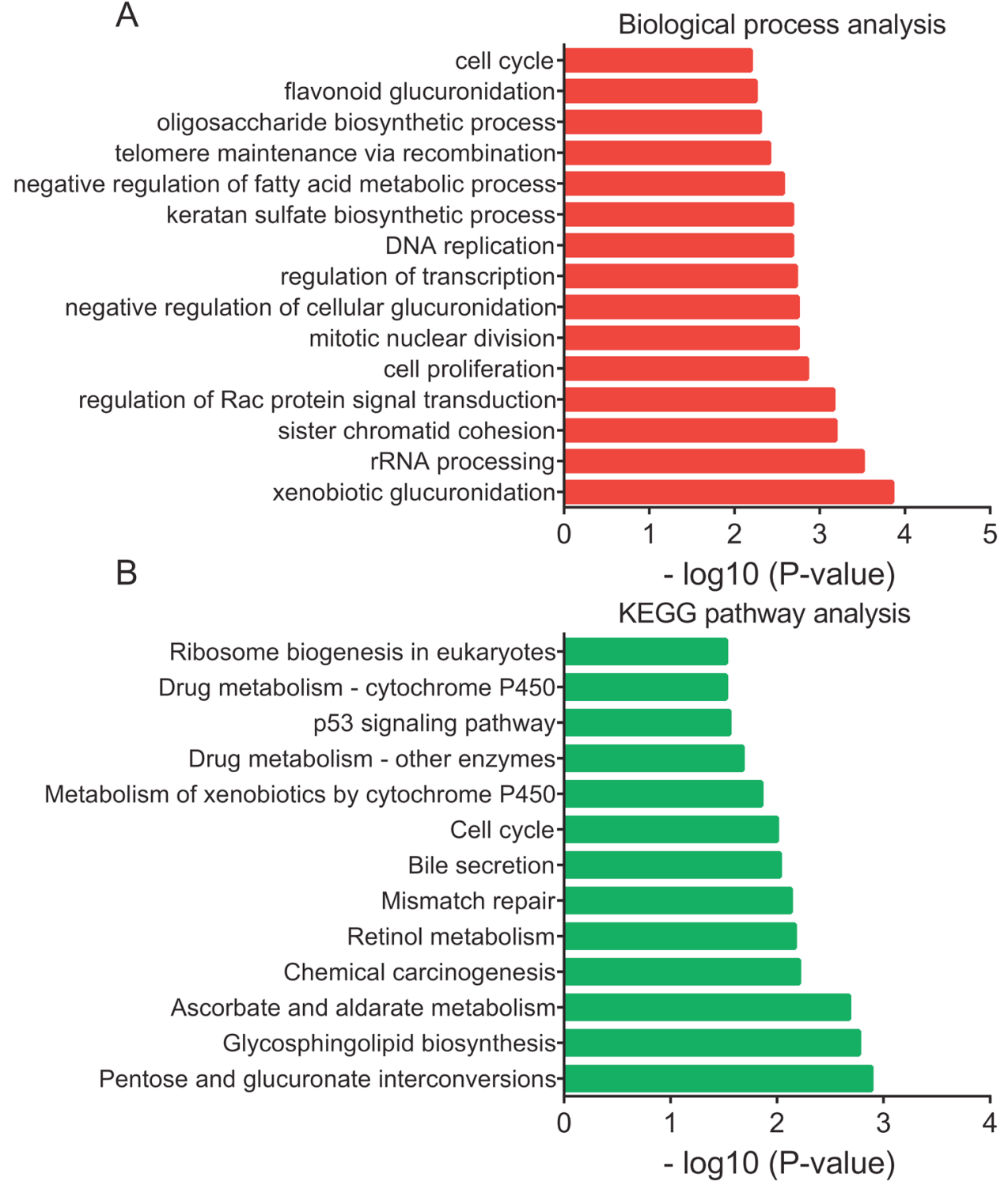

Figure 3. Bioinformatics analysis for differentially expressed lncRNAs in CRC. (A) GO analysis revealed significantly enriched biological processes. (B) KEGG pathway analysis revealed significantly enriched pathways. lncRNA, long non-coding RNA; CRC, colorectal cancer; GO, Gene Ontology; KEGG, Kyoto Encyclopedia of Genes and Genomes.

$(\mathrm{P}=0.0468)$ were associated with an improved overall survival in CRC. These results indicated that the key lncRNAs identified in the current study may serve as biomarkers for CRC.

\section{Discussion}

LncRNAs are cancer progression regulators that are associated with regulating cell proliferation, apoptosis and migration. In CRC, a number of lncRNAs have been demonstrated to serve crucial roles in cancer tumorigenesis. MEG3 has been indicated to regulate the miR-141/PDCD4 axis to affect CRC oxaliplatin resistance (40). DLEU1 has been indicated to promote CRC progression by activating KPNA3 (41). HAND2-AS1 has also been observed to suppress CRC proliferation though sponging miR-1275 (42). However, the expression pattern and functional roles of a large number of lncRNAs remain unclear. In the present study, CRC-associated lncRNAs were identified though re-annotating the GSE21510 dataset. A total of 1,125 upregulated lncRNAs and 1,245 downregulated lncRNAs were identified in CRC compared with normal samples. Among these, a number of lncRNAs, including CRNDE, GAS5, SNHG1 and ZFAS1, were associated with CRC progression. CRNDE has previously been indicated to promote CRC cell proliferation by epigenetically silencing DUSP5/CDKN1A expression (43). GAS5, a well-known lncRNA in a variety of cancer types, suppressed CRC cell proliferation via the miR-182-5p/FOXO3a axis (44). SNHG1 has been demonstrated to serve as an oncogene in CRC though activating the wnt pathway and sponging miR-145 (45). The upregulation of ZFAS1 was associated with poor prognosis and promoted cancer proliferation though sponging miR-484 in CRC (37). However, the functional roles of the majority of lncRNAs remain unclear.

Bioinformatics analysis provides a powerful tool to reveal the potential roles of unknown RNA transcripts in human diseases. LncRNA co-expression networks are used 
A
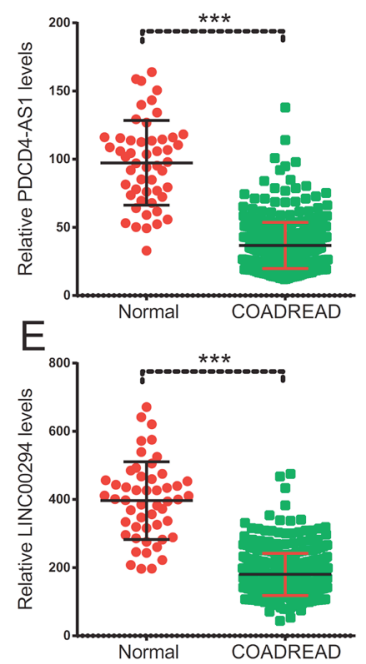

$$
\text { I }
$$

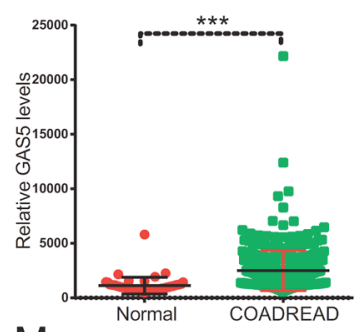

$\mathrm{M}$

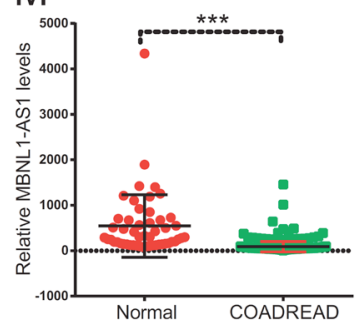

B

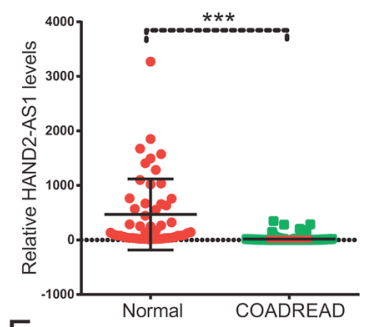

$\mathrm{F}$
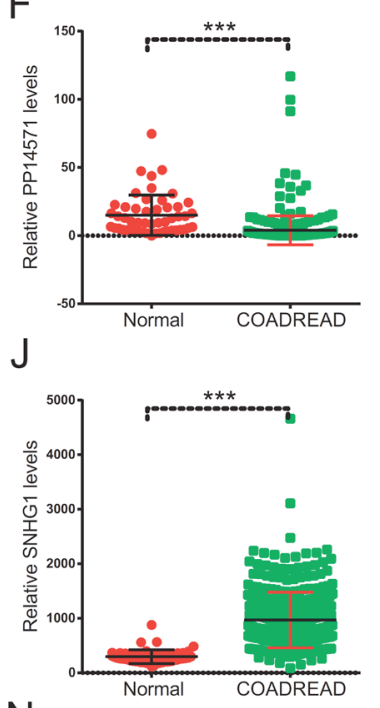

$\mathrm{N}$

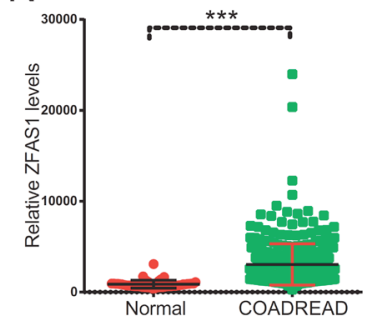

C

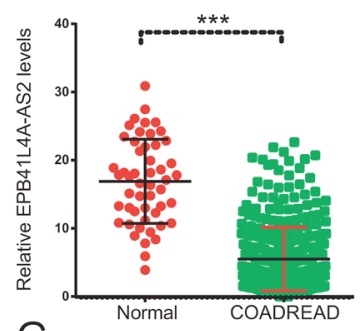

$\mathrm{G}_{1 \mathrm{sen}}$
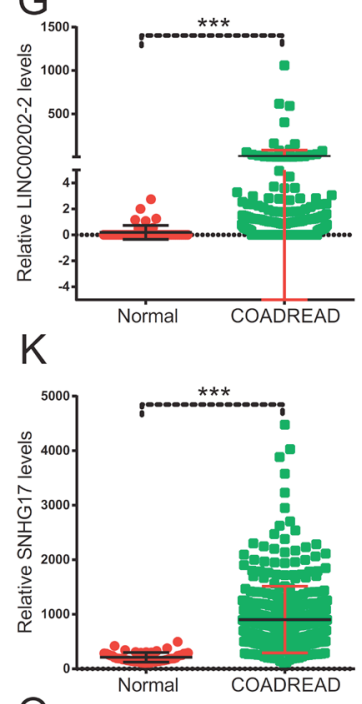

O

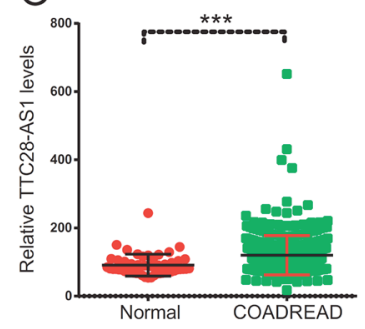

D
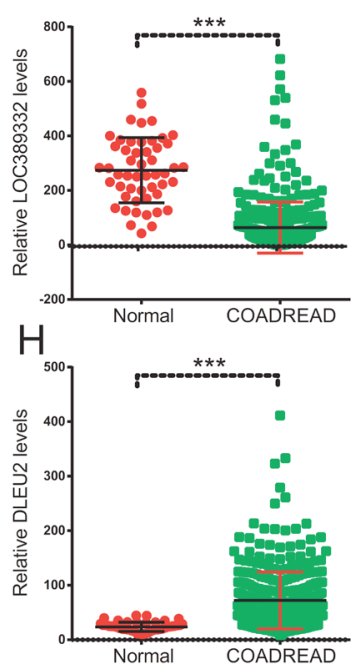

$\mathrm{L}$
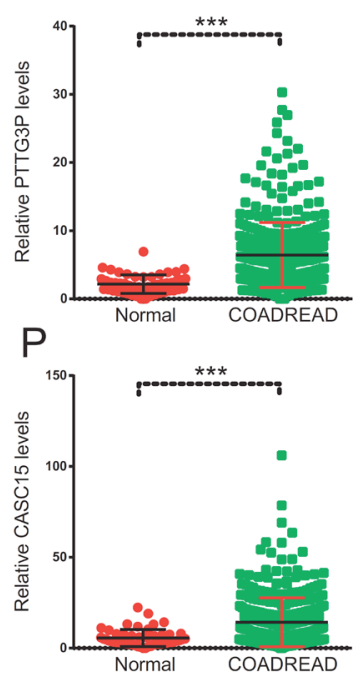

Figure 4. Validation of expression of key lncRNAs in CRC, using the TCGA dataset. The expression levels of (A) PDCD4-AS1,(B) HAND2-AS1,(C) EPB41L4A-AS2, (D) LOC389332, (E) LINC00294 and (F) PP14571 were decreased in CRC compared with normal samples. The expression levels of (G) LINC00202-2, (H) DLEU2, (I) GAS5, (J) SNHG1, (K) SNHG17, (L) PTTG3P, (M) MBNL1-AS1, (N) ZFAS1, (O) TTC28-AS1 and (P) CASC15 were increased in CRC compared with normal tissues. Statistical comparisons between normal and CRC groups were performed using a Mann-Whitney U-test. ${ }^{* * *} \mathrm{P}<0.001$. COAD, colon adenocarcinoma; READ, rectum adenocarcinoma; IncRNA, long non-coding RNA; CRC, colorectal cancer; TCGA, The Cancer Genome Atlas; PDCD4, programmed cell death 4; AS, antisense RNA; HAND2, heart and neural crest derivatives expressed 2; EPB41L4A, erythrocyte membrane protein band 4.1 like 4A; LINC00294, long intergenic non-protein coding RNA 294; DLEU2, deleted in lymphocytic leukemia 2; GAS5, growth arrest specific 5; SNHG1, small nucleolar RNA host gene 1; SNHG17, small nucleolar RNA host gene 17; PTTG3P, pituitary tumor-transforming 3 pseudogene; MBNL1, muscle blind like splicing regulator 1; ZFAS1, ZNFX1 antisense RNA 1; TTC28, tetratricopeptide repeat domain 2B; CASC15, cancer susceptibility 15.

to understand the important roles of lncRNAs in a number of human diseases, including diabetes (46) and major depressive disorder (47). In the present study, differentially expressed lncRNAs co-expression networks were constructed in CRC, which included 47 lncRNAs and 636 mRNAs. The current study indicated that a number of IncRNAs, including LOC389332, LOC101928405, FLJ36848 and EPB41L4A-AS2 were identified as key regulators in CRC and were co-expressed with $>250$ mRNAs in the progression of CRC. LOC389332 was downregulated and associated with favorable prognosis in clear cell renal cell carcinoma (48). EPB41L4A-AS2 was demonstrated to inhibit tumor proliferation and was associated with favorable prognoses in breast cancer and other solid tumors (49). Furthermore, a number of
mRNAs, including YIF1B, ZBTB7B, RUVBL2 and VAMP2, also served an important role in this co-expression network. A previous study indicated that YIF1B was associated with the anterograde traffic pathway and the Golgi architecture (50). ZBTB7B is a transcription factor associated with the regulation of lineage commitment of immature T-cell precursors (51). VAMP2 is a member of the vesicle-associated membrane protein, and VAMP2-NRG1 fusion is a novel oncogenic driver of non-small-cell lung adenocarcinoma (52). Bioinformatics analysis indicated that differentially expressed IncRNAs were associated with 'xenobiotic glucuronidation', 'rRNA processing', 'sister chromatid cohesion', 'regulation of Rac protein signal transduction', 'cell proliferation', 'mitotic nuclear division', 'p53 signaling pathway' and 'cell cycle 

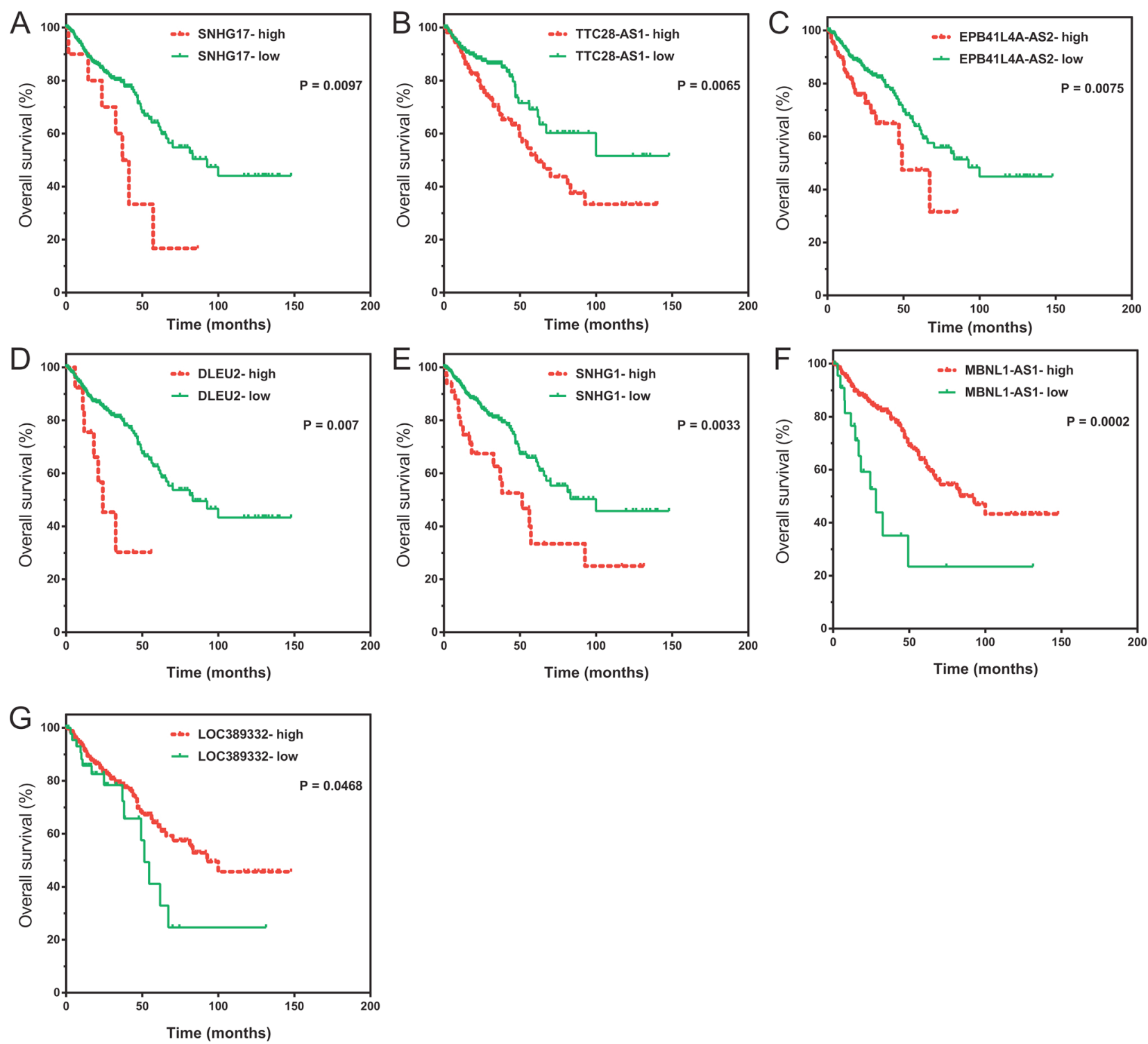

Figure 5. Dysregulation of key lncRNAs is associated with overall survival time in CRC. Kaplan-Meier analysis of the overall survival rates in patients with high and low expression levels of (A) SNHG17, (B) TTC28-AS1, (C) EPB41L4A-AS2, (D) DLEU2, (E) SNHG1, (F) MBNL1-AS1 and (G) LOC389332. Log-rank test was used to examine the significance of the difference between the curves. IncRNA, long non-coding RNA; SNHG17, small nucleolar RNA host gene 17; TTC28, tetratricopeptide repeat domain 2B; AS, antisense RNA; EPB41L4A, erythrocyte membrane protein band 4.1 like 4A; DLEU2, deleted in lymphocytic leukemia 2; SNHG1, small nucleolar RNA host gene 1; MBNL1, muscle blind like splicing regulator 1.

regulation'. Abnormal cell cycle progression is considered a hallmark of human cancer $(53,54)$. Targeting cell cycle checkpoints is a therapeutic approach against cancer (55). A number of compounds were reported as potential anti-cancer drugs in CRC, including itraconazole, a drug which targets cell cycle heterogeneity (56), and 5-Aminosalicylic acid, which inhibits cell cycle progression in a phospholipase D dependent manner (57). The present study revealed that dysregulated IncRNAs in CRC were strongly associated with the regulation of cancer cell cycle progression, indicating that these lncRNAs may serve as novel therapeutic biomarkers for this disease. Previous studies have indicated that the dysregulation of lncRNAs may serve as novel biomarker for a variety of cancers, including CRC. For example, upregulated SNHG6 has been revealed to predict a poor prognosis in CRC (27). In the present study, the prognostic value of key IncRNAs in CRC were evaluated using TCGA CRC dataset PDCD4-AS1,
HAND2-AS1, EPB41L4A-AS2, LOC389332, LINC00294 and PP14571 were observed to be expressed at a low level, and LINC00202-2, DLEU2, GAS5, SNHG1, SNHG17, PTTG3P, MBNL1-AS1, ZFAS1, TTC28-AS1 and CASC15 were indicated to be highly expressed in CRC compared with normal tissues. Furthermore, patients with CRC and higher expression of SNHG17, TTC28-AS1, EPB41L4A-AS2, DLEU2 and SNHG1 exhibited a shorter overall survival time. However, patients with CRC with higher expression of MBNL1-AS1 and LOC389332 exhibited longer overall survival time compared with patients with low expression levels of these genes. These results indicated that high SNHG17, TTC28-AS1, EPB41L4A-AS2, DLEU2 and SNHG1 expression, and low MBNL1-AS1 and LOC389332 expression could predict the negative outcomes of patients with CRC. These analyses, to the best of our knowledge, for the first time, indicated that these lncRNAs may serve as biomarkers for CRC. 
In conclusion, a total of 38 upregulated lncRNAs and 31 downregulated lncRNAs were observed in CRC. Bioinformatics analysis indicated that differentially expressed lncRNAs were associated with 'xenobiotic glucuronidation', 'rRNA processing', 'sister chromatid cohesion', 'regulation of Rac protein signal transduction', 'cell proliferation', 'mitotic nuclear division' and 'cell cycle regulation'. Furthermore, the results revealed that high expression of SNHG17, TTC28-AS1, EPB41L4A-AS2, DLEU2 and SNHG1, and low expression of MBNL1-AS1 and LOC389332 were associated with shorter overall survival time of patients with CRC. The current study provides useful information for identifying novel biomarkers in CRC.

\section{Acknowledgements}

Not applicable.

\section{Funding}

The present study was supported by Youth Research Fund Project of the Health and Family Planning Commission of Nantong (grant no. WQ2015052), the Project for Medical Key Youth Talent of Nantong (grant no. Youth 005), and Nantong Science and Technology Project (grant no. YYZ16043).

\section{Availability of data and materials}

The datasets used and/or analyzed during the current study are available from the corresponding author on reasonable request.

\section{Authors' contributions}

$\mathrm{XC}$ and $\mathrm{BZ}$ conceived and designed the study. $\mathrm{XuZ}, \mathrm{XiZ}$ and LSh developed the methodology. JW, GC, LSh and LSo analyzed and interpreted the data. $\mathrm{XuZ}$ and $\mathrm{XiZ}$ wrote, reviewed and revised the manuscript.

\section{Ethics approval and consent to participate}

Not applicable.

\section{Patient consent for publication}

Not applicable.

\section{Competing interests}

The authors declare that they have no competing interests.

\section{References}

1. Du Z, Sun T, Hacisuleyman E, Fei T, Wang X, Brown M, Rinn JL, Lee MG, Chen Y, Kantoff PW and Liu XS: Integrative analyses reveal a long noncoding RNA-mediated sponge regulatory network in prostate cancer. Nat Commun 7: 10982, 2016.

2. Wang J, Song YX and Wang ZN: Non-coding RNAs in gastric cancer. Gene 560: 1-8, 2015.

3. Wang F, Ying HQ, He BS, Pan YQ, Deng QW, Sun HL, Chen J, Liu X and Wang SK: Upregulated lncRNA-UCA1 contributes to progression of hepatocellular carcinoma through inhibition of miR-216b and activation of FGFR1/ERK signaling pathway. Oncotarget 6: 7899-7917, 2015.
4. Iguchi T, Uchi R, Nambara S, Saito T, Komatsu H, Hirata H, Ueda M, Sakimura S, Takano Y, Kurashige J, et al: A long noncoding RNA, lncRNA-ATB, is involved in the progression and prognosis of colorectal cancer. Anticancer Res 35: 1385-1388, 2015.

5. Liu Y, Zhang M, Liang L, Li J and Chen YX: Over-expression of lncRNA DANCR is associated with advanced tumor progression and poor prognosis in patients with colorectal cancer. Int J Clin Exp Pathol 8: 11480-11484, 2015

6. Cui Y, Zhang F, Zhu C, Geng L, Tian T and Liu H: Upregulated lncRNA SNHG1 contributes to progression of non-small cell lung cancer through inhibition of miR-101-3p and activation of Wnt/ $\beta$-catenin signaling pathway. Oncotarget 8: 17785-17794, 2017.

7. Huarte M: The emerging role of lncRNAs in cancer. Nat Med 21: 1253-1261, 2015.

8. Liu Q, Huang J, Zhou N, Zhang Z, Zhang A, Lu Z, Wu F and Mo YY: LncRNA loc285194 is a p53-regulated tumor suppressor. Nucleic Acids Res 41: 4976-4987, 2013.

9. Shi XF, Sun M, Liu HB, Yao YW and Song Y: Long non-coding RNAs: A new frontier in the study of human diseases. Cancer Lett 339: 159-166, 2013.

10. Hauptman $\mathrm{N}$ and Glavač D: Long non-coding RNA in cancer. Int J Mol Sci 14: 4655-4669, 2013.

11. Zhang H, Chen Z, Wang X, Huang Z, He Z and Chen Y: Long non-coding RNA: A new player in cancer. J Hematol Oncol 6: 37, 2013.

12. Neumann P, Jaé N, Knau A, Glaser SF, Fouani Y, Rossbach O, Krüger M, John D, Bindereif A, Grote $\mathrm{P}$, et al: The lncRNA GATA6-AS epigenetically regulates endothelial gene expression via interaction with LOXL2. Nature Commun 9: 237, 2018.

13. Wang S, Liang K, Hu Q, Li P, Song J, Yang Y, Yao J, Mangala LS, Li C, Yang W, et al: JAK2-binding long noncoding RNA promotes breast cancer brain metastasis. J Clin Invest 127: 4498-4515, 2017.

14. Li C, Li W, Zhang Y, Zhang X, Liu T, Zhang Y, Yang Y, Wang L, Pan H, Ji J and Wang C: Increased expression of antisense lncrna SPINT1-AS1 predicts a poor prognosis in colorectal cancer and is negatively correlated with its sense transcript. Onco Targets Ther 11: 3969-3978, 2018.

15. Zhang E, Li W, Yin D, De W, Zhu L, Sun S and Han L: c-Myc-regulated long non-coding RNA H19 indicates a poor prognosis and affects cell proliferation in non-small-cell lung cancer. Tumor Biol 37: 4007-4015, 2016.

16. Sostres C, Gargallo CJ and Lanas A: Aspirin, cyclooxygenase inhibition and colorectal cancer. World World J Gastrointest Pharmacol Ther 5: 40-49, 2014.

17. Marley AR and Nan H: Epidemiology of colorectal cancer. Int J Mol Epidemiol Genet 7: 105-114, 2016.

18. Ma S, Yang D, Liu Y, Wang Y, Lin T, Li Y, Yang S, Zhang W and Zhang R: LncRNA BANCR promotes tumorigenesis and enhances adriamycin resistance in colorectal cancer. Aging (Albany NY) 10: 2062-2078, 2018.

19. Li G, Wang C, Wang Y, Xu B and Zhang W: LINC 00312 represses proliferation and metastasis of colorectal cancer cells by regulation of miR-21. J Cell Mol Med 22: 5565-5572, 2018.

20. Zhang K, Liu J, Li C, Peng X, Li H and Li Z: Identification and validation of potential target genes in papillary thyroid cancer. Eur J Pharmacol 843: 217-225, 2019.

21. Yuan W, Li X, Liu L, Wei C, Sun D, Peng S and Jiang L: Comprehensive analysis of lncRNA-associated ceRNA network in colorectal cancer. Biochem Biophys Res Commun 508: 374-379, 2019.

22. Miao Y, Sui J, Xu SY, Liang GY, Pu YP and Yin LH: Comprehensive analysis of a novel four-lncRNA signature as a prognostic biomarker for human gastric cancer. Oncotarget 8: 75007-75024, 2017.

23. Wang S, Ke H, Zhang H, Ma Y, Ao L, Zou L, Yang Q, Zhu H, Nie J, Wu C and Jiao B: LncRNA MIR100HG promotes cell proliferation in triple-negative breast cancer through triplex formation with p27 loci. Cell Death Dis 9: 805, 2018.

24. Wang X, Li M, Wang Z, Han S, Tang X, Ge Y, Zhou L, Zhou C, Yuan Q and Yang M: Silencing of long noncoding RNA MALAT1 by miR-101 and miR-217 inhibits proliferation, migration, and invasion of esophageal squamous cell carcinoma cells. J Biol Chem 290: 3925-3935, 2015.

25. Cao Q, Wang N, Qi J, Gu Z and Shen HJ: Long non-coding RNA-GAS5 acts as a tumor suppressor in bladder transitional cell carcinoma via regulation of chemokine (C-C motif) ligand 1 expression. Mol Med Rep 13: 27-34, 2016. 
26. Zhang X, Sun S, Pu JK, Tsang AC, Lee D, Man VO, Lui WM, Wong ST and Leung GK: Long non-coding RNA expression profiles predict clinical phenotypes in glioma. Neurobiol Dis 48: $1-8,2012$.

27. Tsukamoto $\mathrm{S}$, Ishikawa $\mathrm{T}$, Iida $\mathrm{S}$, Ishiguro $\mathrm{M}$, Mogushi $\mathrm{K}$, Mizushima H, Uetake H, Tanaka H and Sugihara K: Clinical significance of osteoprotegerin expression in human colorectal cancer. Clin Cancer Res 17: 2444-2450, 2011.

28. Binefa G, Rodríguez-Moranta F, Teule A and Medina-Hayas M: Colorectal cancer: From prevention to personalized medicine. World J Gastroenterol 20: 6786-6808, 2014.

29. R Core Team. R: A language and environment for statistical computing. R Foundation for Statistical Computing, Vienna, Austria, 2013.

30. Gautier L, Cope L, Bolstad BM and Irizarry RA: Affy-analysis of Affymetrix GeneChip data at the probe level. Bioinformatics 20: 307-315, 2004

31. Golubeva Y and Rogers K: Collection and preparation of rodent tissue samples for histopathological and molecular studies in carcinogenesis. Methods Mol Biol 511: 3-60, 2009.

32. Graham LD, Pedersen SK, Brown GS, Ho T, Kassir Z, Moynihan AT, Vizgoft EK, Dunne R, Pimlott L, Young GP, et al: Colorectal neoplasia differentially expressed (CRNDE), a novel gene with elevated expression in colorectal adenomas and adenocarcinomas. Genes Cancer 2: 829-840, 2011

33. Mourtada-Maarabouni M, Hedge VL, Kirkham L, Farzaneh F and Williams GT: Growth arrest in human T-cells is controlled by the non-coding RNA growth-arrest-specific transcript 5 (GAS5). J Cell Sci 121: 939-946, 2008.

34. Zhang H, Zhou D, Ying M, Chen M, Chen P, Chen Z and Zhang F. Expression of long non-coding RNA (lncRNA) small nucleolar RNA Host Gene 1 (SNHG1) exacerbates hepatocellular carcinoma through suppressing miR-195. Med Sci Monit 22: 4820-4829, 2016.

35. Ying Z, Sun L, Xuan L, Pan Z, Li K, Liu S, Huang Y, Zhao X, Huang L, Wang Z, et al: Reciprocal changes of circulating long non-coding RNAs ZFAS1 and CDR1AS predict acute myocardial infarction. Sci Rep 6: 22384, 2016.

36. Zhao Y, Qin ZS, Feng Y, Tang XJ, Zhang T and Yang L: Long non-coding RNA (lncRNA) small nucleolar RNA host gene 1 (SNHG1) promote cell proliferation in colorectal cancer by affecting P53. Eur Rev Med Pharmacol Sci 22: 976-984, 2018.

37. Xie S, Ge Q, Wang X, Sun X and Kang Y: Long non-coding RNA ZFAS1 sponges miR-484 to promote cell proliferation and invasion in colorectal cancer. Cell Cycle 17: 154-161, 2018

38. Liu F, Gao H, Li S, Ni X and Zhu Z: Long non-coding RNA ZFAS1 correlates with clinical progression and prognosis in cancer patients. Oncotarget 8: 61561-61569, 2017.

39. Budczies J, Klauschen F, Sinn BV, Győrffy B, Schmitt WD, Darb-Esfahani S and Denkert C: Cutoff finder: A Comprehensive and straightforward web application enabling rapid biomarker cutoff optimization. PLoS One 7: e51862, 2012.

40. Li M, Bian Z, Yao S, Zhang J, Jin G, Wang X, Yin Y and Huang Z: Up-regulated expression of SNHG6 predicts poor prognosis in colorectal cancer. Pathol Res Pract 214: 784-789, 2018.

41. Liu T, Han Z, Li H, Zhu Y, Sun Z and Zhu A: LncRNA DLEU1 contributes to colorectal cancer progression via activation of KPNA3. Mol Cancer 17: 118, 2018.

42. Zhou J, Lin J, Zhang H, Zhu F and Xie R: LncRNA HAND2-AS1 sponging miR-1275 suppresses colorectal cancer progression by upregulating KLF14. Biochem Biophys Res Commun 503: $1848-1853,2018$
43. Ding J, Li J, Wang H, Tian Y, Xie M, He X, Ji H, Ma Z, Hui B, Wang $\mathrm{K}$ and Ji G: Long noncoding RNA CRNDE promotes colorectal cancer cell proliferation via epigenetically silencing DUSP5/CDKN1A expression. Cell Death Dis 8: e2997, 2017.

44. Cheng K, Zhao Z, Wang G, Wang J and Zhu W: lncRNA GAS5 inhibits colorectal cancer cell proliferation via the miR-182-5p/FOXO3a axis. Oncol Rep 40: 2371-2380, 2018.

45. Tian T, Qiu R and Qiu X: SNHG1 promotes cell proliferation by acting as a sponge of miR-145 in colorectal cancer. Oncotarget 9: 2128-2139, 2017.

46. Wang P, Fu H, Cui J and Chen X: Differential lncRNA-mRNA co-expression network analysis revealing the potential regulatory roles of lncRNAs in myocardial infarction. Mol Med Rep 13: 1195-1203, 2016

47. Egede LE and Zheng D: Independent factors associated with major depressive disorder in a national sample of individuals with diabetes. Diabetes Care 26: 104-111, 2003.

48. Jin P, Wang J and Liu Y: Downregulation of a novel long non-coding RNA, LOC389332, is associated with poor prognosis and tumor progression in clear cell renal cell carcinoma. Exp Ther Med 13: 1137-1142, 2017.

49. Xu S, Wang P, You Z, Meng H, Mu G, Bai X, Zhang G, Zhang J and Pang D: The long non-coding RNA EPB41L4A-AS2 inhibits tumor proliferation and is associated with favorable prognoses in breast cancer and other solid tumors. Oncotarget 7: 20704-20717, 2016.

50. Alterio J, Masson J, Diaz J, Chachlaki K, Salman H, Areias J, Al Awabdh S, Emerit MB and Darmon M: Yif1B is involved in the Anterograde traffic pathway and the Golgi Architecture. Traffic 16: 978-993, 2015.

51. Wang L, Wildt KF, Castro E, Xiong Y, Feigenbaum L, Tessarollo $\mathrm{L}$ and Bosselut R: The zinc finger transcription factor Zbtb7b represses CD8-lineage gene expression in peripheral CD4+ T cells. Immunity 29: 876-887, 2008.

52. Jung Y, Yong S, Kim P, Lee HY, Jung Y, Keum J, Lee S, Kim J and Kim J: VAMP2-NRG1 fusion gene is a novel oncogenic driver of non-small-cell lung adenocarcinoma. J Thorac Oncol 10: 1107-1111, 2015.

53. Scaggiante B, Dapas B, Bonin S, Grassi M, Zennaro C, Farra R, Cristiano L, Siracusano S, Zanconati F, Giansante C and Grassi G: Dissecting the expression of EEF1A1/2 genes in human prostate cancer cells: The potential of EEF1A2 as a hallmark for prostate transformation and progression. Br J Cancer 106: 166-173, 2012

54. Hanahan D and Weinberg RA: Hallmarks of cancer: The next generation. Cell 144: 646-674, 2011.

55. Visconti R, Della Monica R and Grieco D: Cell cycle checkpoint in cancer: A therapeutically targetable double-edged sword. J Exp Clin Cancer Res 35: 153, 2016.

56. Buczacki SJA, Popova S, Biggs E, Koukorava C, Buzzelli J, Vermeulen L, Hazelwood L, Francies H, Garnett MJ and Winton DJ: Itraconazole targets cell cycle heterogeneity in colorectal cancer. J Exp Med 215: 1891-1912, 2018.

57. Baan B, Dihal AA, Hoff E, Bos CL, Voorneveld PW, Koelink PJ, Wildenberg ME, Muncan V, Heijmans J, Verspaget HW, et al: 5-Aminosalicylic acid inhibits cell cycle progression in a phospholipase D dependent manner in colorectal cancer. Gut 61: 1708-1715, 2012.

This work is licensed under a Creative Commons Attribution-NonCommercial-NoDerivatives 4.0 International (CC BY-NC-ND 4.0) License. 